\title{
Utilizando o Modelo ADDIE para o Desenvolvimento e Avaliação de um Processo Educacional Inspirado na Educação 4.0
}

\author{
Deivid Eive Silva ${ }^{1}$, Marialina Corrêa Sobrinho ${ }^{2}$, Natasha Malveira C. Valentim ${ }^{1}$ \\ ${ }^{1}$ Universidade Federal do Paraná (UFPR), Curitiba - PR - Brasil \\ ${ }^{2}$ Universidade Federal do Oeste do Pará (UFOPA), Santarém - PA - Brasil \\ \{dessilva@inf.ufpr.br, linasobrinho@gmail.com, natasha@inf.ufpr.br\}
}

\begin{abstract}
Education 4.0 is a student-centered learning paradigm that seeks to prepare them for the contemporary world. Therefore, this paper presents the use of the stages of the ADDIE model (Analyze, Design, Develop, Implement, and Evaluate) for the conception and evolution of the Teacher Assistance Educational Process (TAEP4.0). TAEP4.0 aims to support teachers in the preparation of classes based on the context of Education 4.0. TAEP4.0 also encourages the development of 21st-Century competencies and skills, such as problem-solving, critical thinking, and creativity. To this end, TAEP4.0 encourages to use of the technological resources available in the school.
\end{abstract}

Resumo. A Educação 4.0 é um paradigma de aprendizagem centrado no estudante que busca prepará-los para o mundo contemporâneo. Portanto, este artigo apresenta o uso das etapas do modelo ADDIE (Analisar, Projetar, Desenvolver, Implementar e Avaliar) para a concepção e evolução do Teacher Assistance Educational Process (TAEP4.0). O TAEP4.0 objetiva apoiar os professores na preparação de aulas baseadas no contexto da Educação 4.0. O TAEP4.0 também incentiva o desenvolvimento de competencias e habilidades do Século XXI, como resolução de problemas, pensamento crítico e criatividade. Para este fim, o TAEP4.0 incentiva o uso dos recursos tecnológicos disponíveis na escola.

\section{Introdução}

O processo de informatização e digitalização da indústria, também conhecido como Indústria 4.0 ou quarta Revolução Industrial, impactou vários setores da sociedade, incluindo o setor educacional. Desse modo, percebeu-se a necessidade de uma formação mais alinhada ao mundo contemporâneo que permita melhorar os resultados acadêmicos, ajudar os estudantes a desenvolverem autorregulação, no qual o estudante estrutura, monitora e avalia o seu próprio aprendizado sem intervenção externa [Ganda e Boruchovitch, 2018], além de reduzir os conflitos entre estudantes, melhorar a disciplina na sala de aula, auxiliar jovens a terem relacionamentos mais saudáveis e bem-sucedidos na escola e na vida [Korbel e Paulus, 2017].

Neste contexto, percebeu-se que os recursos tecnológicos podem ter uma boa contribuição na educação das pessoas. $\mathrm{Na}$ era 4.0 acredita-se que somente pessoas capacitadas poderão controlar as tecnologias digitais. Portanto, é indicado que os jovens sejam capacitados para lidar com esses recursos. Mas também, se faz importante ensiná- 
los sobre os valores associados ao uso desses recursos com responsabilidade, ética e integridade [Angrisani et al., 2018]. Essa prática poderá engajá-los na aprendizagem curricular, prepará-los com as competências e habilidades importantes para a vida no Século XXI, sem perder a dimensão e os significados dos processos de ensino e aprendizagem [Abed, 2016]. Assim, espera-se que no futuro, os jovens possuam a capacidade de desenvolver tecnologia e processos, mas também saibam quando, onde e como usar esses recursos. Esse tipo de pensamento é reflexivo e interdisciplinar e pode impactar positivamente a escola e a indústria [Angrisani et al., 2018]. Este é um novo paradigma que busca redefinir o modelo de educação, conhecido como Educação 4.0.

Este trabalho tem como objetivo apresentar o uso do modelo ADDIE (Analyse, Design, Develop, Implement, Evaluate) para o desenvolvimento e avaliação de um processo educacional de apoio ao professor da Educação Básica inspirado na Educação 4.0, chamado Teacher Assistance Educacional Process (TAEP4.0). O ADDIE possui as seguintes etapas: (1) Analyse (Analisar): nesta etapa foram investigados na literatura conceitos e possibilidades de como trabalhar a Educação 4.0 na prática; (2) Design (Projetar): nesta etapa foram organizados e definidos os passos do TAEP4.0 no formato de diagrama com o propósito de apoiar os professores nos desafios da Educação 4.0, como a inserção de computação no currículo escolar; (3) Develop (Desenvolver): nesta etapa foram pensados no uso e na interação do TAEP4.0 por professores da Educação Básica. Desse modo, o TAEP4.0 foi produzido como PDF clicável e instrucional contendo links para recursos e materiais de apoio; (4) Implement (Implementar): nesta etapa, o TAEP4.0 foi refinado e atualizado por meio de um estudo exploratório com educadores, incluindo especialistas em Informática na Educação; e (5) Evaluate (Avaliar): nesta etapa foi verificada a contribuição do TAEP4.0 para os professores e estudantes por meio de um estudo de caso e um estudo exploratório com gestoras educacionais. Esta última etapa do ADDIE (Avaliar) permitiu a evolução do TAEP4.0 para sua versão website, o qual é apresentada como parte dos resultados deste artigo, juntamente com o detalhamento dos procedimentos de avaliação.

O TAEP4.0 incentiva o desenvolvimento de competências e habilidades do Século XXI, tais como: pensamento computacional, criatividade, tomada de decisão, pensamento crítico, trabalho em equipe, autonomia, dentre outras. O TAEP4.0 busca: a) aumentar a interação e o conhecimento dos professores sobre a Educação 4.0 ; b) subsidiar o trabalho do professor por meio da inserção da Computação no currículo escolar; c) fazer com que os recursos tecnológicos disponíveis na escola sejam utilizados; d) permitir aulas interativas e práticas por meio de uma aprendizagem baseada em projeto; e e) possibilitar o protagonismo do estudante [Silva, 2020].

Este trabalho está organizado em 6 Seções. Na Seção 2 serão apresentados os principais conceitos da Educação 4.0 e alguns trabalhos relacionados que usaram o ADDIE para preparação de materiais e recursos. Na Seção 3 será mostrado como o modelo ADDIE apoiou o desenvolvimento do TAEP4.0. Na Seção 4 será descrito o funcionamento do TAEP4.0 e apresentada a sua versão website. Na Seção 5 serão realizadas as discussões. Por fim, na Seção 6 serão apresentadas as considerações finais e próximos passos desta pesquisa.

\section{Background}

O conceito de Educação 4.0 surge com o propósito de preparar os estudantes para o mundo contemporâneo. $\mathrm{Na}$ Educação 4.0, espera-se que o processo de aprendizagem 
seja personalizado, no qual os estudantes tenham flexibilidade para serem os responsáveis pela construção do seu conhecimento com liberdade de atingir os seus objetivos [Hartono et al., 2018]. Além disso, busca-se uma mentalidade dinâmica para que os estudantes possam resolver problemas de forma colaborativa e criativa, criar soluções inovadoras e tecnológicas, e serem sujeitos ativos e responsáveis por sua aprendizagem. Desse modo, a Educação 4.0 busca ensinar mais do que conteúdos, suscitando o desenvolvimento de competências e habilidades do Século XXI.

$\mathrm{Na}$ Educação 4.0, muito se discute sobre as competências e habilidades do Século XXI. Segundo Messias et al. [2018], elas são consideradas importantes para a vida no Século XXI e inovadoras para viver na era da Educação 4.0. Portanto, é importante entender as definições dessas terminologias e, como elas estão relacionadas. Conforme o Centro Europeu de Formação Profissional [CEDEFOP, 2008], a Habilidade é a capacidade de executar tarefas e resolver problemas, enquanto a Competência é a capacidade de aplicar esses resultados da aprendizagem em distintos contextos, como educação, trabalho, desenvolvimento pessoal ou profissional. As competências e habilidades mais estudadas nos trabalhos relacionados identificados na literatura são: a) criatividade e inovação; b) resolução de problemas; c) comunicação; d) colaboração, e e) aprender a aprender [Messias et al., 2018].

Uma das maneiras encontradas para reunir os conceitos da Educação 4.0, foi por meio do uso do modelo ADDIE (Analyse, Design, Develop, Implement, Evaluate), que é considerado um processo sistemático que apoia designers instrucionais, desenvolvedores de conteúdo e professores a criar design de ensino eficiente e eficaz [Aldoobie, 2015]. O modelo ADDIE é um dos mais utilizados na literatura e permitiu identificar evidências sobre Educação 4.0. A Subseção a seguir apresenta trabalhos que foram identificados numa busca manual sobre o uso desse modelo.

\subsection{Trabalhos Relacionados}

Neste sentido, algumas iniciativas sobre o uso do ADDIE para apoiar o processo de aprendizagem no contexto da Educação 4.0 foram identificados na literatura. As iniciativas e as etapas utilizadas são descritas a seguir:

- Unidade Instrucional (UI) para o ensino da Computação [Cruz Alves et al., 2016]: Os autores utilizaram o ADDIE para a criação de uma UI de forma multidisciplinar que permite integrar alguns conceitos práticos de programação com os conteúdos da disciplina de História para estudantes do Ensino Fundamental. Na etapa Analisar, inicialmente, foram estudados os perfis dos estudantes, o ambiente escolar e definidos os objetivos de aprendizagem. $\mathrm{Na}$ etapa Projetar, os pesquisadores elaboraram a UI a fim de fazer o ensino da Computação ser envolvente e motivador aos estudantes. Na etapa Desenvolver, vários materiais instrucionais foram desenvolvidos, como guia do instrutor, jogos demonstrativos, rubrica com os critérios de avaliação e o questionário de avaliação. $\mathrm{Na}$ etapa Implementar, a UI foi aplicada com estudantes do $5^{\circ}$ e $7^{\circ}$ ano do Ensino Fundamental utilizando o Scratch. Por fim, na etapa Avaliar, os autores verificaram os aspectos relacionados a UI para o ensino de conceitos de computação no Ensino Fundamental por meio do questionário de avaliação.

- Aplicativo de Aprendizagem Móvel [Karim et al., 2018]: Os pesquisadores utilizaram o ADDIE para o desenvolvimento de uma aplicação mobile para estudantes de graduação de uma Universidade da Malásia. O ADDIE motivou 5 processos, tais como: (1) revisar a análise do uso do telefone móvel entre os 
estudantes, (2) projetar o aplicativo de aprendizagem móvel, (3) desenvolver os aplicativos de aprendizagem mobile para o curso de Redação, (4) implementar os módulos de aprendizagem móvel, e (5) avaliar os aplicativos de aprendizagem móvel. Neste estudo, apenas o processo 1 (etapa Analisar) foi apresentado. A etapa 1 teve o objetivo de identificar as perspectivas e práticas da aprendizagem móvel entre estudantes, a partir dos componentes de uso, tipos e tempo gasto no uso de dispositivos móveis.

- Realistic Mathematics Education (RME) [Hikayat et al., 2020]: Os pesquisadores se limitaram as etapas Analisar e Projetar do ADDIE, a fim de elaborar materiais de ensino para uma Educação Matemática Realista e melhorar habilidades de Pensamento Crítico. Os participantes foram estudantes do $7^{\circ}$ ano do Ensino Fundamental de uma escola pública da Indonésia. Na etapa Analisar, os conteúdos curriculares da matemática foram analisados. Na etapa Projetar, um módulo foi apresentado, sendo composto por capa, prefácio, mapa conceitual, competências essenciais, competências básicas e indicadores, realização de competências, índice, atividades do módulo, avaliação e resumo. Os pesquisadores preveem continuar o estudo com o desenvolvimento, estágios de implementação e avaliação da aprendizagem.

Esta seção apresentou três diferentes maneiras de usar o ADDIE, como o desenvolvimento de unidades instrucionais, materiais instrucionais e aplicativos móveis. Dos trabalhos identificados, apenas Cruz Alves et al., [2016] consideraram as 5 etapas do ADDIE. Além disso, apenas Karim et al., [2018] fazem menção ao termo Educação 4.0. Para este artigo, as etapas do ADDIE foram utilizadas de forma integral para o desenvolvimento e avaliação do TAEP4.0 inspirado na Educação 4.0.

\section{Uso do Modelo ADDIE para o desenvolvimento e Avaliação do TAEP4.0}

O TAEP4.0 foi produzido com base no modelo ADDIE. As etapas foram discutidas e organizadas com a finalidade de atender os problemas, as motivações e os objetivos apresentados neste artigo. Desse modo, foi possível entender e definir os passos do TAEP4.0 para apoiar professores na preparação de aulas e projetos educacionais no contexto da Educação 4.0.

\subsection{Contexto de Uso}

O TAEP4.0 foi produzido originalmente para apoiar os professores da Educação Básica, especificamente Ensino Fundamental e Médio, onde este processo foi usado. Porém, os passos do TAEP4.0 podem ser utilizados por professores de outros níveis de ensino como Técnico, Profissionalizante e Superior. O TAEP4.0 pode ser direcionado a quaisquer disciplinas como Português, Artes, Biologia, Espanhol, Geografia, História, Química, dentre outras. Neste sentido, o TAEP4.0 incentiva o trabalho interdisciplinar permitindo aos professores integrar a sua disciplina com as demais, utilizando as áreas do termo STEAM (um acrônimo em inglês para as áreas Ciências, Tecnologia, Engenharia, Artes e Matemática). Por fim, o professor define as competências e habilidades que necessita incentivar nos seus estudantes ao longo dos projetos.

\subsection{Desenvolvimento e Avaliação}

As etapas do ADDIE utilizadas para concepção e avaliação do TAEP4.0 são detalhadas a seguir: 
$\mathrm{Na}$ etapa Analisar, um Mapeamento Sistemático da Literatura (MSL) foi conduzido, onde foram realizadas buscas automáticas e manuais sobre Educação $4.0 \mathrm{em}$ 5 bases, sendo SCOPUS, ACM, IEEExplore, e anais do SBIE e RBIE [Silva et al., 2021]. Paralelamente, quatro estudos preliminares foram realizados com diferentes tecnologias educacionais escolhidas por professores de diferentes instituições de ensino, sendo da Educação Básica e Ensino Superior. Estes professores foram escolhidos por conveniência. As tecnologias foram: a) Storytelling Digital (contação de histórias por meio da criação de curta-metragem) e STEAM (para incentivar o trabalho interdisciplinar) com estudantes da $3^{\mathrm{a}}$ série do Ensino Médio [Silva et al., 2019a]; b) Programação em Blocos (utilizando o Scratch) e Espiral do Saber (para apoiar o aprender fazendo na criação de jogos educativos) com estudantes do $5^{\circ}$ ano do Ensino Fundamental [Silva et al., 2019b]; c) Computação Desplugada (ensino da lógica de programação por meio de desafios matemáticos) com estudantes do $4^{\circ}$ ao $9^{\circ}$ ano do Ensino Fundamental [Silva et al., 2020a]; e d) Robótica Educacional (projetos de robôs físicos, simuladores e drones) com estudantes de graduação em Ciência da Computação e pós-graduação em Informática [Silva et al., 2020b]. Em todos os estudos preliminares foram trabalhadas competências e habilidades do Século XXI, como criatividade e resolução de problemas, e conhecimentos que se aplicam à Educação 4.0. Essas tecnologias educacionais foram identificadas no MSL como emergentes para o contexto da Educação 4.0. Os estudos preliminares tiveram o propósito de perceber as necessidades dos estudantes em sala de aula e experimentar tecnologias educacionais da Educação 4.0, a fim de ter condições de propor algo nesse contexto.

$\mathrm{Na}$ etapa Projetar, os passos do TAEP4.0 foram definidos por meio de um diagrama baseado nas evidências identificadas nos estudos preliminares, como a prática em sala de aula e experiência no uso de tecnologias educacionais, e com base no MSL, que permitiu verificar iniciativas relacionadas à Educação 4.0. Das iniciativas identificadas, foram selecionadas aquelas que apresentam atividades práticas e processo metodológico que apoia o desenvolvimento das competências e habilidades do Século XXI. A partir da análise desses estudos, algumas práticas foram organizadas e adicionadas ao TAEP4.0, tais como: a) personalização de conteúdo; b) incentivo de experiências e conexões entre estudantes; c) incentivo de competências e habilidades do Século XXI; e d) inserção da Computação e Informática no currículo escolar.

$\mathrm{Na}$ etapa Desenvolver, o TAEP4.0 foi produzido originalmente para uso de professores da Educação Básica. O TAEP4.0 está organizado em três atividades principais, sendo: Planejamento (o professor prepara a sua aula), Execução (o professor executa o seu planejamento com os estudantes), e Verificação (o professor conduz o processo de avaliação formativa com os estudantes). Além disso, o TAEP4.0 é composto por 13 passos, distribuídos nas atividades de Planejamento, Execução e Verificação das competências e habilidades desenvolvidas. Na etapa Desenvolver, o TAEP4.0 foi produzido no formato de PDF clicável para facilitar o uso e a interação dos professores com os passos por meio de links para acesso a recursos e materiais de apoio.

$\mathrm{Na}$ etapa Implementar, o TAEP4.0 foi usado de forma parcial por quatro especialistas em Informática na Educação de uma escola de Educação Básica, um professor de Computação de uma escola de Ensino Médio Técnico, e um professor de Geografia de Ensino Superior. Os participantes foram orientados a planejar um projeto utilizando os sete passos de Planejamento do TAEP4.0. São eles: Definir Escopo, Verificar os Recursos Tecnológicos, Preparar Avaliação Diagnóstica, Elaborar os Desafios Problemas, Definir o Projeto, Preparar os Materiais de Apoio e Organizar o 
Projeto. Assim, os participantes experimentaram o TAEP4.0 parcialmente, pois eles não conseguiram aplicar os projetos elaborados e nem verificar o impacto desses projetos junto aos estudantes. Durante este estudo, os participantes leram as instruções e os exemplos apresentados no TAEP4.0 com a finalidade de elaborar um projeto. Ao final, os participantes entregaram o planejamento do seu projeto e responderam seu grau de aceitação em relação ao TAEP4.0 por meio de um questionário pós-uso baseado nos indicadores do Modelo de Aceitação de Tecnologia (TAM3) [Silva et al., 2020c].

Na etapa Avaliar, o TAEP4.0 foi experimentado em sua totalidade por quatro professoras da Educação Básica para elaboração e realização de aulas com o uso dos recursos tecnológicos, e teve a participação de 415 estudantes. Portanto, capacitação e estudos foram conduzidos com o TAEP4.0 no Colégio Dom Amando em Santarém-PA, em parceria com a coordenação de Tecnologias Educacionais desta instituição de ensino. Essas atividades tiveram uma duração de 3 meses. Seguindo o TAEP4.0, as professoras elaboraram projetos para $\mathrm{o} 1^{\mathrm{o}}$ bimestre com base nos conteúdos programáticos das disciplinas que lecionam. A realização dos projetos buscou respeitar o calendário escolar, sendo ajustados aos horários de aula das professoras e horários disponíveis no laboratório de Informática. Ao final dos projetos, os estudantes responderam um questionário de autoavaliação sobre as competências e habilidades desenvolvidas nos projetos. Além disso, as professoras responderam um questionário sobre suas experiências utilizando o TAEP4.0 e percepções obtidas durante o processo de orientação e acompanhamento dos estudantes nos projetos. Após este estudo de caso com professoras e estudantes, o TAEP4.0 foi organizado e disponibilizado como website. Esta última versão do TAEP4.0 foi submetida a avaliação de duas gestoras educacionais da Secretária Municipal de Educação (SEMED) de Manaus. O estudo com as gestoras foi realizado devido as suas experiências com formação de professores e elaboração de iniciativas a serem adotadas nas escolas de Educação Básica. Este estudo exploratório foi organizado em três etapas: a) Implementação: apresentação da abordagem do estudo para as gestoras; b) Avaliação: as gestoras fizeram suas anotações numa ficha avaliativa, dando suas sugestões de melhorias, e registrando dúvidas e questionamentos; e c) Entrevista: as gestoras participaram de uma entrevista semiestruturada para coletar as suas percepções durante o uso e avaliação do TAEP4.0 [Silva et al., 2020d]. Finalmente, o TAEP4.0 foi atualizado para uma versão mais recente, o qual será apresentada na Seção a seguir.

\section{Resultado}

O TAEP4.0 tem o objetivo de apoiar os professores na elaboração de aulas no contexto da Educação 4.0. Portanto, o TAEP4.0 incentiva o uso dos recursos tecnológicos para trabalhar competências e habilidades do Século XXI. O TAEP4.0 pode ser acessado por meio do seguinte endereço eletrônico https://sites.google.com/view/taep/home. O vídeo demonstrativo do TAEP4.0 está disponível no link https://youtu.be/Hpv-TxrN6y0. Os passos do TAEP4.0 foram definidos a partir das evidências identificadas nos estudos preliminares e MSL (etapa Analisar do ADDIE).

O processo foi organizado seguindo o modelo BPMN (Business Process Model and Notation) com objetivo de estruturar o fluxo de trabalho dos professores. O BPMN foi escolhido, pois é um dos mais utilizados na literatura para o desenvolvimento de processos [Pereira, 2011]. O TAEP4.0 é composto por treze passos divididos em três atividades principais, sendo atividade de Planejamento (o que professor precisa realizar antes da aula): Definir Escopo, Verificar Recursos Tecnológicos, Preparar Avaliação 
Diagnóstica, Definir Desafios Problemas, Definir Projeto, Preparar Materiais de Apoio, Organizar Projeto; atividade de Execução (o que professor realiza durante a aula): Aplicar Avaliação Diagnóstica, Aplicar Projeto, Aprender Fazendo e Preparar Avaliação Formativa); e atividade de Verificação (representa o dinamismo durante as aulas por meio da relação entre professor e estudantes): Conduzir Avaliação Formativa, Refletir e Compartilhar. Estes passos são apresentados no diagrama da Figura 1. O TAEP4.0 prevê a omissão e/ou inserção de passos conforme a necessidade do professor e realidade da escola. $\mathrm{O}$ detalhamento de cada passo do TAEP4.0 está disponível em Silva et al. (2020c).

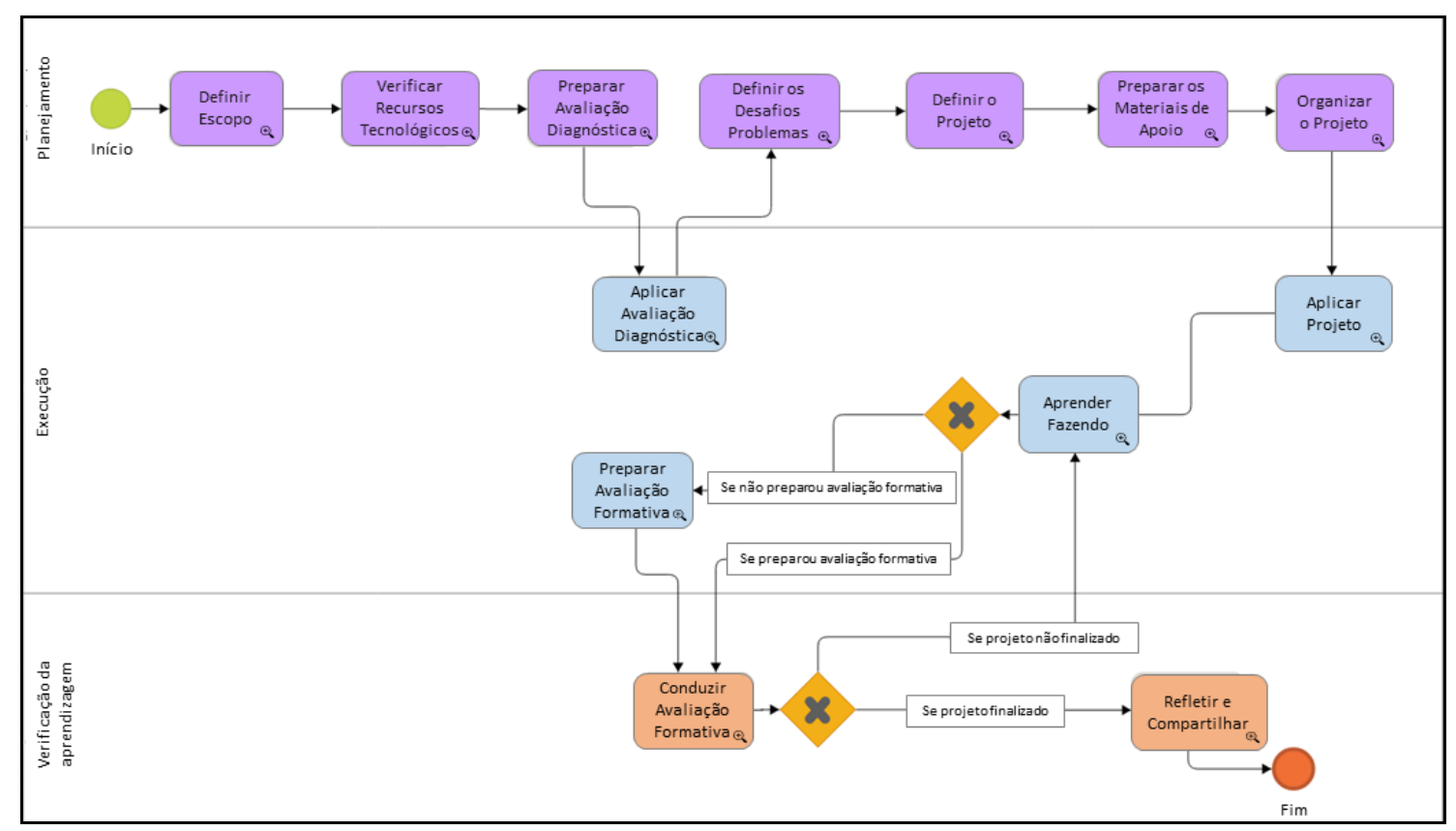

Figura 1. Processo Educacional TAEP4.0 [Silva et al., 2020c]

No TAEP4.0, alguns passos foram postos de maneira estratégica para interligar as atividades e tornar o fluxo o mais natural possível para o professor. As variações entre uma atividade e outra são chamadas de transições. Portanto, os passos Preparar e Aplicar Avaliação Diagnóstica permitem a primeira transição do processo (entre as atividades de Planejamento e Execução), onde o professor inicialmente verifica o que está a sua disposição como recursos e materiais. A partir disso, ele identifica as necessidades dos estudantes, tendo condições de ajustar e finalizar o seu planejamento de forma mais adequada e próximo da realidade dos estudantes. Posteriormente, ele organiza e propõe o projeto, o que caracteriza a segunda transição (entre as atividades de Planejamento e Execução). No TAEP4.0, é dado ênfase para o passo Aprender Fazendo, pois é onde há a participação ativa do estudante. O passo Aprender Fazendo colabora para uma maior relação entre professor e estudantes, provocando uma série de transições entre as atividades de Execução e Verificação. Esse dinamismo é representado por meio de condicionais que indicam que enquanto os estudantes estiverem produzindo, o professor realiza o acompanhamento e fornece as orientações necessárias. As orientações são finalizadas quando os estudantes concluem os projetos.

Os passos do TAEP4.0 contêm exemplos de uso, notas explicativas e ferramentas, que poderão ajudar o professor a elaborar uma aula no contexto da Educação 4.0. De modo geral, o TAEP4.0 funciona da seguinte forma: o professor 
desenvolve uma atividade a partir de um documento de escopo disponível no próprio TAEP4.0. Mediante este documento, são definidas a turma e a disciplina, de que forma poderá trabalhar a interdisciplinaridade na sala de aula e quais habilidades poderá incentivar nos estudantes. Posteriormente, o professor escolhe a tecnologia conforme a realidade da sua escola. Dentre as opções de tecnologias, alguns exemplos de atividades desplugadas ou com materiais recicláveis foram disponibilizados para que o professor possa elaborar um projeto mesmo com poucos recursos financeiros. Além disso, recursos são apresentados para que o professor possa trabalhar com programação em blocos, gamificação, realidade aumentada, dentre outros (Figura 2).

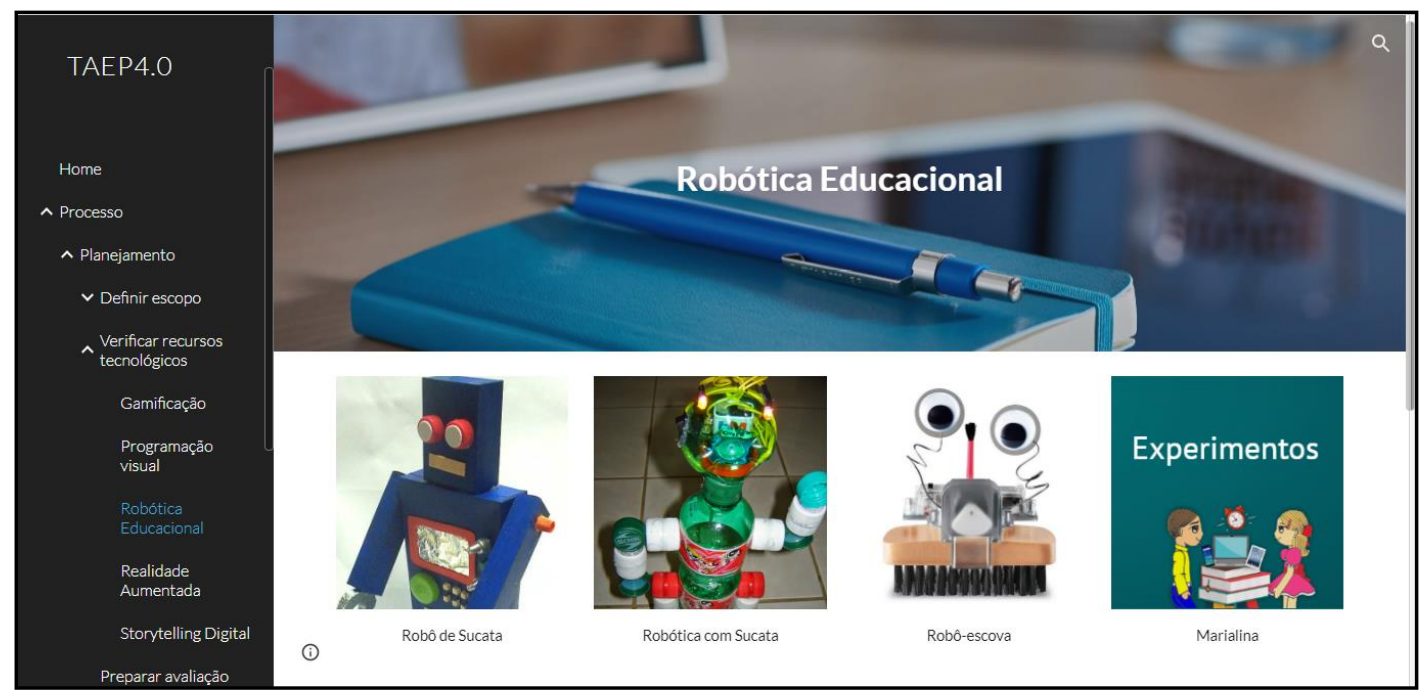

Figura 2. Tela Robótica Educacional

\section{Discussões}

O ADDIE é um dos modelos de Design Instrucional mais utilizados na literatura. Por meio deste artigo, identificou-se que o ADDIE também está sendo adotado para desenvolver tecnologias no contexto da Educação 4.0. Tendo como base os trabalhos citados na subseção 2.1, percebeu-se que o ADDIE: a) possibilitou a criação de uma UI para apoiar o ensino da computação por meio do Scracth no Ensino Fundamental [Cruz Alves et al., 2016]; b) ajudou a identificar perspectivas e práticas a serem implementadas nas tecnologias [Karim et al., 2018]; e c) permitiu adaptar as tecnologias conforme as características dos participantes [Hikayat et al., 2020].

Nesse sentido, o modelo ADDIE contribuiu para o desenvolvimento e avaliação do TAEP4.0. Por meio das etapas do ADDIE, planejou-se as atividades necessárias. Assim, primeiramente, foi possível experimentar algumas tecnologias educacionais da Educação 4.0, a fim de ter condições de indicar algo nesse contexto, ainda considerado recente. Paralelamente, realizou-se um MSL que permitiu identificar literatura relevante sobre o termo Educação 4.0 e as competências e habilidades do Século XXI. Esse conjunto de evidências retornados na etapa Analisar do ADDIE possibilitou projetar e desenvolver o TAEP4.0 com a finalidade de apoiar os professores nos novos desafios provocados pela Educação 4.0, como preparar os estudantes para o mundo contemporâneo. Além disso, o ADDIE permitiu trazer profissionais para dentro do desenvolvimento, como especialistas em Informática na Educação (etapa Implementar), o que contribuiu para fazer os ajustes necessários e evoluir o TAEP4.0, antes de ser utilizado no contexto real de uso. Posteriormente, o ADDIE possibilitou usar o 
TAEP4.0 com professoras, estudantes e gestoras (etapa Avaliar), o que contribuiu para ele ser utilizado de forma integral, sendo possível evoluir para a sua versão website.

\section{Considerações Finais e Trabalhos Futuros}

Este artigo teve o objetivo de apresentar o uso do ADDIE como apoio para o desenvolvimento do TAEP4.0 (Teacher Assistance Educational Process). O ADDIE como modelo de Design Instrucional tem o propósito de criar design de ensino eficiente e eficaz. Portanto, o uso do ADDIE foi interessante para este trabalho, pois permitiu desenvolver um processo que apoia o professor na preparação de atividades como aulas e projetos no contexto da Educação 4.0. Nesse sentido, o ADDIE motivou uma série de estudos experimentais que envolveram especialistas em Informática na Educação, professores de variadas disciplinas e níveis de ensino, e gestoras educacionais.

O TAEP4.0 incentiva o uso dos recursos tecnológicos para desenvolver competências e habilidades do Século XXI, como pensamento computacional, criatividade e trabalho em equipe. O TAEP4.0 foi desenvolvido com a tecnologia Google Sites (uma ferramenta gratuita e estruturada que possibilita o desenvolvimento de páginas web de forma colaborativa). O TAEP4.0 permite inserir computação no currículo escolar a partir de aulas práticas, interativas e diversificadas. Seguindo os passos do TAEP4.0, o professor seleciona as informações e a tecnologia apropriada conforme a realidade da escola, a disciplina e as necessidades dos estudantes. Para isso, em cada etapa do TAEP4.0, foram apresentados alguns materiais disponíveis na web, a título de sugestão, com o intuito de agilizar e facilitar a criação dos projetos.

Como última etapa do ADDIE (avaliar), pretende-se continuar avaliando e evoluindo o TAEP4.0, para que ele possa ajudar os professores no planejamento das suas aulas no formato 4.0. Dessa maneira, pretende-se transformar o website TAEP4.0 em um sistema funcional que permita uma maior interação e tomada de decisões pelos professores na escolha dos elementos, tendo a possibilidade de omitir e incluir passos que considere pertinente a sua aula, conforme a realidade da escola e necessidades dos estudantes. Por fim, pretende-se incluir mais materiais e recursos no TAEP4.0 para ser disponibilizado de forma gratuita para as escolas de Educação Básica.

\section{Referências}

Abed, A. L. Z. (2016). O desenvolvimento das habilidades socioemocionais como caminho para a aprendizagem e o sucesso escolar de estudantes da educação básica. Construção psicopedagógica, 24(25), p. 8-27, 2016.

Aldoobie, N. (2015). Addie model. American International Journal of Contemporary Research, 5 (6), p. 68-72, 2015.

Angrisani, L., Arpaia, P., Capaldo, G., Moccaldi, N., Salatino, P., Ventre, G. (2018). Academic Fablab at University of Naples Federico II: New Research and Development Opportunities in the Fileds of IoT and Industry 4.0. Journal of Physics: Conference Series, v. 1065, 2018.

CEDEFOP - Centro Europeu de Formação Profissional. (2008). Terminology of European education and training policy. A selection of 100 key terms. Luxembourg: Office for Official Publications of the European Communities.

Cruz Alves, N., Rodrigues, P. E., Borgatto, A. F., Gresse von Wangenheim, C., Hauck, J. C. R. (2016). Ensino de Computação de Forma Multidisciplinar em Disciplinas de 
História no Ensino Fundamental--Um Estudo de Caso. Revista Brasileira de Informática na Educação, 24(3), doi: 10.5753/RBIE.2016.24.03.31

Ganda, D. R., Boruchovitch, E. (2018). A Autorregulação da Aprendizagem: Principais Conceitos e Modelos Teóricos. Psic. da Ed., São Paulo, 46 (1), pp. 71-80, doi: $10.5935 / 2175-3520.20180008$

Hartono, S., Kosala, R., Supangkat, S., e Ranti, B. (2018). Smart Hybrid Learning Framework Based on Three-Layer Architecture to Bolster Up Education 4.0. In International Conference on ICT for Smart Society (ICISS), pp. 1-5. doi: 10.1109/ICTSS.2018.8550028

Hikayat, S., Yahya \& Hery. (2020). Design of Realistic Mathematics Education Approach Improve Critical Thinking Skills. Universal Journal of Educational Research. 8(6): 2232-2244.

Karim, R. A., Abu, A. G., Adnan, A. H. M., Suhandoko, A. D. J. (2018). The use of mobile technology in promoting Education 4.0 for higher education. Advanced Journal of Technical and Vocational Education, 2(3), p. 34 - 39, 2018.

Korbel, V., Paulus M. (2017). Do Teaching Practices Impact Socio-emotional Skills?. IES Working Paper 4/2017. IES FSV. Charles University, 2017.

Messias, G., Rodrigues, U., Braga, L., Nakamura, W., Ferreira, B., Paiva, A., Valentim, N. (2018). Education 4.0 and 21st Century Skills: A Case Study with Robotics Activities in Classroom. In: XXIX Brazilian Symposium on Computers in Education. p. 715-724, doi: http://dx.doi.org/10.5753/cbie.sbie.2018.715

Pereira, M. F. (2011). Modelo de produção de material didático: O uso da notação BPMN em curso a distância. Revista de Administração e Inovação, 8(4), p. 45-66.

Silva, D., Corrêa Sobrinho, M., Valentim, N. (2019a). STEAM and Digital Storytelling: a case study with high school students in the context of Education 4.0. In SBIE 2019, pp. 159 - 168, doi: http://dx.doi.org/10.5753/cbie.sbie.2019.159

Silva, D., Corrêa Sobrinho, M., Valentim, N. (2019b). Criação de Jogos Educacionais para apoiar o Ensino da Matemática: um Estudo de Caso no Contexto da Educação 4.0. In WIE 2019, doi: http://dx.doi.org/10.5753/cbie.wie.2019.1179

Silva, D., Corrêa Sobrinho, M., Valentim, N. (2020a). Educação 4.0: um Estudo de Caso com Atividades de Computação Desplugada na Amazônia Brasileira. In COTB 2020, pp. 1 - 7, doi: https://doi.org/10.14210/cotb.v11n1.p141-147

Silva, D., Sousa, A., Oliveira, M., Corrêa Sobrinho, M., Todt, E., Valentim, N. (2020b). Education 4.0: Robotics Projects to Encourage 21st Century Skills. In RENOTE 2021, pp. 450 - 459, doi: https://doi.org/10.22456/1679-1916.110285

Silva, D., Corrêa Sobrinho, M., Valentim, N. (2020c). TAEP4.0: Teacher Assistance Educational Process to Promote 21st Century Skills in the Context of Education 4.0. In CSEDU 2020, pp. 249-259, doi: 10.5220/0009387702490259

Silva, D. (2020d). TAEP4.0: Teacher Assistance Educational Process Based in the Context of Education 4.0. Dissertação de mestrado, Programa de Pós-graduação em Informática - Universidade Federal do Paraná. Curitiba, pp. 163, doi: 10.13140/RG.2.2.35858.17603

Silva, D., Lopes, T., Corrêa Sobrinho, M., Valentim, N. (2021). Investigating Initiatives to Promote the Advancement of Education 4.0. In CSEDU 2021, pp. 458-466, doi: $10.5220 / 0010439704580466$ 